\title{
The rise of genetically engineered mouse models of pancreatitis: A review of literature
}

https://doi.org/10.1515/bmc-2018-0011

received July 12, 2018; accepted October 19, 2018.

Abstract: Pancreatitis is increasingly recognized as not merely a local inflammation of the pancreas but also a disease with high frequency of systemic sequelae. Current understanding of the cellular mechanisms that trigger it and affect the development of sequelae are limited. Genetically engineered mouse models can be a useful tool to study the pathophysiology of pancreatitis. This article gives an overview of the genetically engineered mouse models that spontaneously develop pancreatitis and discusses those that most closely replicate different pancreatitis hallmarks observed in humans.

Keywords: genetically engineered mouse models; pancreatitis; inflammation; autophagy; endoplasmic reticulum stress.

\section{Introduction}

Acute pancreatitis has a global incidence of 34 cases per 100000 population and chronic pancreatitis - 9 cases per 100000 population[1]. Emerging studies have shown that acute, recurrent acute, and chronic pancreatitis may represent a disease continuum[2]. Furthermore, there is a growing appreciation of the effect of pancreatitis on development of metabolic disorders, such as exocrine pancreatic insufficiency (EPI), post-pancreatitis diabetes mellitus (PPDM), and ectopic fat accumulation in the pancreas [3-6]. Currently the natural course of pancreatitis and the pathogenesis of the resulting sequelae are poorly understood. As such this review summaries current

\footnotetext{
*Corresponding author: Maxim S. Petrov, Department of Surgery, Faculty of Medical and Health Sciences, The University of Auckland, Auckland, New Zealand, E-mail: max.petrov@gmail.com Troy L. Merry: Maurice Wilkins Centre for Molecular Biodiscovery, The University of Auckland, Auckland, New Zealand; Discipline of Nutrition, Faculty of Medical and Health Sciences, The University of Auckland, Auckland, New Zealand
}

genetically engineered mouse models (GEMM) that may be used to study the pancreatitis phenotype, with particular focus on those that show hallmarks of the humans.

\section{Pancreatitis in humans and mice}

While there are numerous aetiological factors, the overwhelming majority of pancreatitis is either biliary or alcohol-related. The severity of acute pancreatitis varies from mild uncomplicated disease to critical disease associated with both local and systemic complications. A large body of evidence has demonstrated that the two key determinants of severity in acute pancreatitis are organ failure (absent, transient, or persistent) and pancreatic complications (absent, non-infectious, or infectious). Determinants-based classification of the severity of acute pancreatitis (composed of four categories - mild, moderate, severe, and critical) is useful for the clinical assessment of severity in individual patients and for comparing groups of patients $[7,8]$. The distinct advantages of this new classification is that it uses widely accepted and unambiguous terms, it can be applied in both early and late phases of acute pancreatitis, it can facilitate communication between treating physicians, and it promotes standardization for management of acute pancreatitis [9]. Although significant overall progress has been made during the last few decades (evidenced by a reduction in mortality rates), the improved outcomes have not been due to any treatments based on specific pathophysiology. A wide range of drugs has been evaluated in randomized controlled trials and have proved ineffective in the treatment of acute pancreatitis[10]. These include aprotinin, atropine, calcitonin, fresh frozen plasma, glucagon, gabexate, glucocorticoids, lexipafant, nonsteroidal anti-inflammatory drugs, and octreotide.

Combining what we have learnt from clinical studies with mouse models of pancreatitis is a potentially powerful approach to identify effective pathogentic preventative and treatment options. There are several advantages to using mouse experimental approaches to 
test hypothesis generated from clinical investigations. Causative relationships between molecular signalling mechanisms or environmental stressor can more easily be investigated through interventions in tightly controlled environments, such as pharmacologic treatments, diets, genetic modifications. Inbreed mice strains are isogenic so mechanisms of action can be isolated with little consideration of individual genetic variability. Most importantly, mouse models allow a ready access to tissues not easily obtained in the clinic. This is becoming more important, with increasing evidence suggesting that the pathophysiology of pancreatitis is not localised to the pancreas [11, 12]. Ultimately the effectiveness of a murine model to test preclinical therapeutics is dependent on how well it characterises the human phenotype.

\section{Non-GEMM of pancreatitis}

Common mouse pancreatitis models involve repetitive treatments with exogenous stimuli that induce cellular damage and result in impaired pancreatic function and include caerulein, lipopolysaccharides, arginine, ethanol, chlorine deficient ethionine supplemented diet and trinitorbenzene [13]. The relative ease of implementation and low cost of such models, compared to developing a GEMM mouse, has made them a popular choice. Of these stimuli, the most widely used and well-characterised is caerulein - an ortholog of the gastrointestinal hormone cholecystokinin (CCK). When administered as a high single or repeated dose caerulein induces mild reversible pancreatitis. Depending on the administration strategy employed this can resemble many of the common characteristics of human pancreatitis, including aberrant zymogen activation in the acinar cell, impaired exocrine pancreatic secretion, inflammation, morphological abnormalities, fibrosis and trans-differentiation of acini $[13,14]$. However, pancreatitis in humans is not associated with elevated CCK levels, and whether human acinar cells express CCK receptors is also not unclear [15]. The inherent differences in the specificity and pathogenesis process of chemical-induced pancreatitis in rodents compared to human pancreatitis may question the physiological relevance of such models. While more technically challenging models (such as surgical obstruction of the pancreatic duct) provides a more pathophysiological stimulus, they may only be applicable to human pancreatitis cases resulting directly from obstructions [14].

An alternative approach that may be more specific to the underlying pathophysiology causing pancreatitis is the adoption of genetic mouse models. Manipulation of genes associated with the development and pathophysiology of pancreatitis has the potential to model clinical pancreatitis and provide insight into the underlying molecular signalling pathways. With a wide spread adoption of CRISPR/Cas9 technology to make transgenic mice in relatively short time frames and refinement of tissue and temporal targeted Cre/lox system, it is anticipated that the use of transgenic mouse models to study pancreatitis will increase in the coming years.

While non-GEMM models of pancreatitis have proven to be, and will continue to be valuable models for investigating the underlining mechanisms of pancreatitis, the sections below will provide an overview of currently available GEMM pancreatitis models. For a more comprehensive review covering both non-GEMM models and the more popular GEMM models of pancreatitis, readers are referred to the 2018 review by Klauss et al. [16]. For the remainder of this review we have limited ourselves to the models that spontaneously develop pancreatitis symptoms and do not develop pancreatic adenocarcinoma at a young age.

\section{GEMM of pancreatitis}

There have been three major types of genetic manipulations used in pancreatitis models: 1) traditional or global genetic knockout, which involves disruption of a genes sequence so the related protein can no longer be translated in any of the animals tissues, 2) insertion of a transgene or alteration of the genes coding sequence to induce increased translation of a given protein (transgenic overexpression), and 3) both of these approaches can be used in a time and/or tissue specific manor, often through the Cre/lox recombinase technology. In this approach, a pair of short sequences (called loxP sites) are inserted into the genome, flanking a gene (or part thereof) of interest. In theory, the loxP sites do not affect gene function until a Cre recombinase enzyme is introduced to recombine them and, depending on the target sequence, this can activate, repress, or exchange part of the gene of interest that is flanked. There are a large number of Cre lines that use drivers that are predominantly expressed in the pancreas, meaning that this technology can be used to specifically alter gene expression in different pancreatic cell types at different stages of development and independent of most other tissues [17]. Some of the most popular drives in pancreatitis research have been $P d x 1$ (which targets prepancreatic endoderm), Ptf1a (which targets 
both prepancreatic endoderm and acinar cells), and the elastase promotor (which targets acinar cells only). To allow temporal control of recombination, the Cre recombinase has been coupled with a mutated version of the estrogen receptor hormone-binding domain [18], which can only enter the nucleus and recombine the loxP sites following tamoxifen treatment. While this approach means that multiple tamoxifen treatments are often required to induce complete Cre recombination, it has the distinct advantage of being able to avoid phenotypes that result primarily from developmental defects or are masked by compensatory adaptations that occur during development.

Table 1 provides an overview of some of the current GEMM that spontaneously develop pancreatitis-like phenotypes. These models can be broadly grouped into target pathways that include attempts to replicate genetic variants related to hereditary types of pancreatitis and related diseases, alter pathological mechanisms associated with the pancreatitis such as inflammation, endoplasmic reticulum (ER) stress and autophagy, or models that have shown a pancreatitis-like phenotype as a result of the other developmental or signalling pathways in the pancreas or systemically. Below we discuss some of the models that most closely resemble human disease.

\section{Trypsinogen hereditary models}

Classic hereditary pancreatitis is associated with gainof-function mutation in the cationic trypsinogen gene (protease serine 1) PRSS1 [19], with the two most common being R122H and N29I [20]. Trypsinogen is the precursor for the digestive enzyme trypsin and is produced by the pancreatic acinar cells. The premature or unregulated activation of trypsinogen has been hypothesised to induce auto-digestion of the pancreas, initiating secondary events such as inflammatory and cellular death that drive the development of pancreatitis. To test this hypothesis several groups have either overexpressed the human or rodent PRSS1 gene, or inserted a PRSS1 transgene mutated at R122H or N29I, which causes trypsinogen autoactivation [21-23]. When induced prenatally mice spontaneously develop mild symptoms of pancreatitis such as early anomaly vacuolisation that gets progressively worse with age resulting in widespread pancreatic inflammation, fibrosis and lipid infiltration similar to hereditary pancreatitis in humans. However, recent reports suggest that the expression of human PRSS1 may be toxic to mouse pancreas, potentially limiting the usefulness of this model [24].
Under normal circumstances acinar cells co-produce PSTI (pancreatic secretory trypsin inhibitor), also known as SPINK1 (Serine Protease Inhibitor Kazal-type 1), which inhibits the activity of trypsin in the pancreas. Therefore, an alternative model to assess the role of autodigestion in pancreatitis development is to inactivate SPINK1. Indeed, there appears to be a relationship between SPINK1 mutations in humans and development of pancreatitis $[25,26]$. Furthermore, global ablation of the mouse SPINK1 homolog, SPINK3, elevates acinar trypsin activity and results in acinar cell authophagy without regeneration and death within two weeks of birth [27]. While SPINK3 is expressed in other tissues (including the kidney and lung), this phenotype can be rescued by acinar specific overexpression of PSTI [28], indicating that the pancreatic autophagy and early death primarily results from pancreas dysfunction. However, these mice do not show the traditional hallmarks of human pancreatitis, such as pancreatic inflammation and apoptosis, suggesting that SPINK3 deletion-induced pancreatitis may be more representative of an early developmental defect rather progressive human pancreatitis.

Interestingly, a geenticc mutations in the CPA1 gene are over-represented in paediatric pancreatitis [29] and knocking in a N256K point mutation in this gene in mice results in spontaneous and progressive development of chronic pancreatitis [30]. The CPA1 gene encodes for the digestive enzyme carboxypeptidase $\mathrm{A} 1$, which is a monomeric pancreatic exopeptidase involved in the cleavage of amino acids. The pancreatic phenotype of these mice is characterised by many of the hallmarks of human chronic pancreatitis, including pancreatic atrophy, fibrosis, and inflammatory cell infiltration that may result from protein misfolding associated with ER stress. The above facts, coupled with a very a targeted genetic approach (single point mutation), makes this model a very attractive one to study pancreatitis.

While genetic manipulation of trypsinogen-related genes is a good model for studying the pathogenesis of hereditary pancreatitis, the vast majority of hereditary pancreatitis cases develop prenatally in the absence of identified or common genetic variants. To investigate the role of trypsinogen activation in post-natal pancreatitis, Gaiser et al. [23] used the elastase CreERT2 system to rapidly turn on the PRSS1 gene (trypsinogen activated) in acinar cells after birth [23]. These mice showed a more severe pancreatic phenotype and high rates of mortality. This effect was attenuated when increases in trypsinogen expression was induced more slowly, suggesting that there are mechanisms in place to compensate for prenatal and/or slow increases in trypsinogen. However, when 


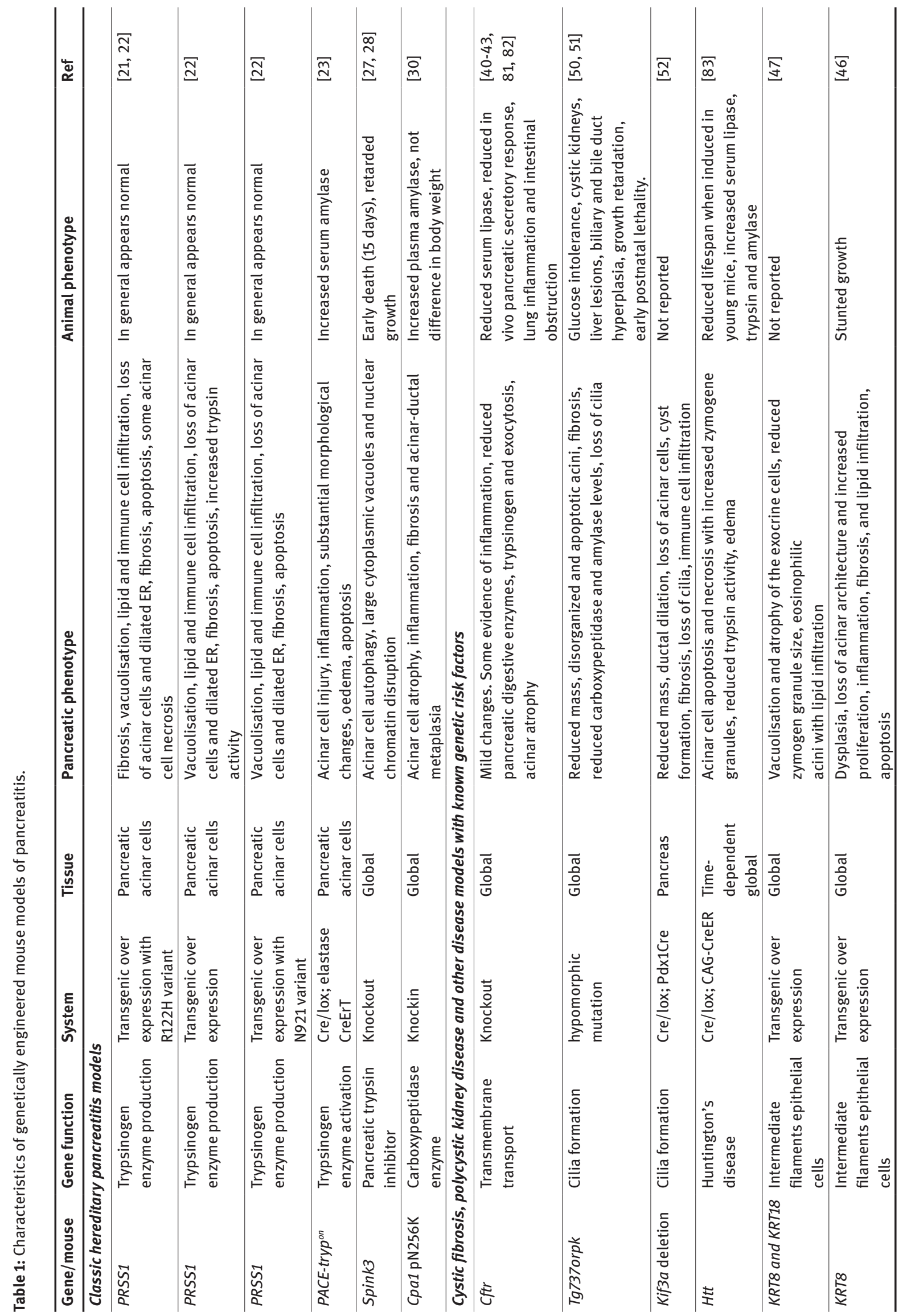




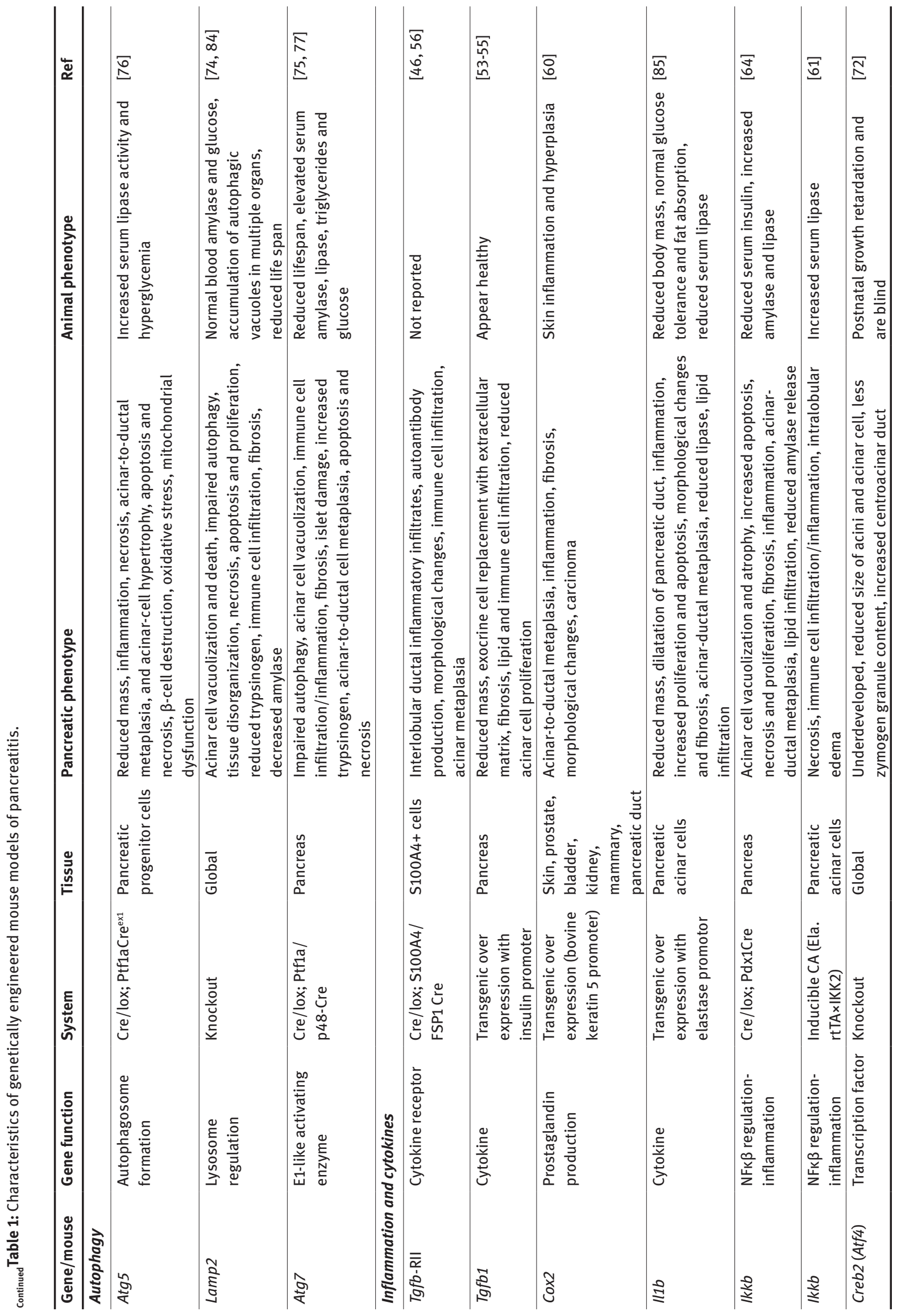




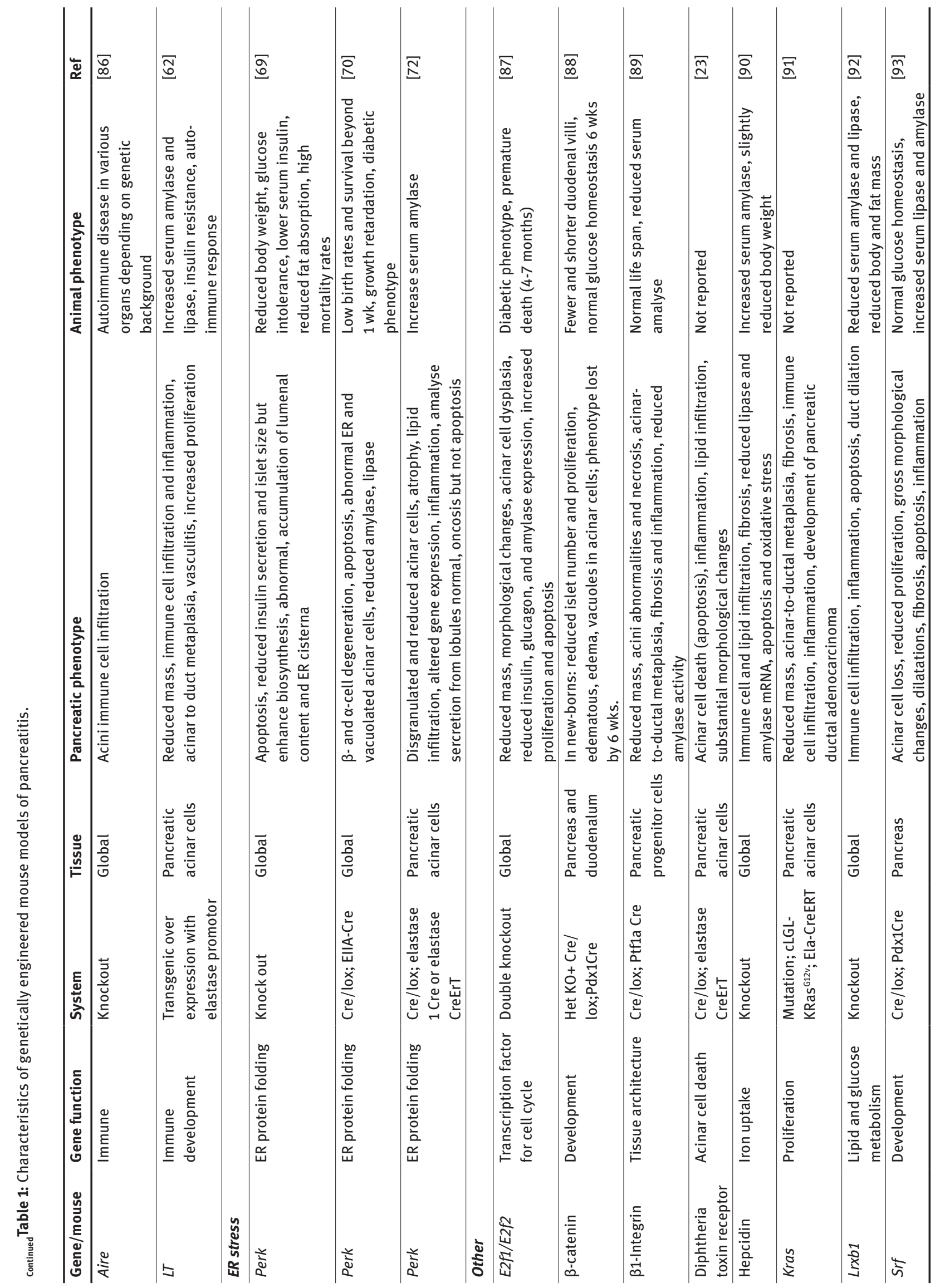


these processes are overwhelmed, such as during aging or with rapid increase in trypsinogen expression, then pancreatitis develops.

The model of adult inducible and acinar cell trypsinogen activation [23] may serve as a helpful model of both acute and chronic pancreatitis in mice, however further detailed in vivo phenotyping needs to be undertaken. It would be of interest to investigate whether potential pancreatitis risk factors like aging or obesity alter their phenotype, and whether this mouse model develops systemic hallmarks of pancreatitis such as impaired glucose homeostasis or inflammation. This will have a potential to reduce the burden of pancreatitis associated with obesity [31, 32].

\section{Genetic disease models that present with pancreatitis}

Trypsinogen gene mutations are commonly associated with hereditary pancreatitis, however other diseases with an identified genetic components (such as cystic fibrosis [33-35], polycystic kidney disease [36], and the liver dysfunction associated with mutations in keratin genes [37, 38]) often develop a pancreatitis-like phenotypes. This could suggest these genetic variations directly, or indirectly through their related pathologies, can influence pancreatic function. Mutations in the cystic fibrosis transmembrane conductance regulator (CFTR) gene that controls chloride ion channel function causes dysregulation of epithelial fluid transport characteristic of cystic fibrosis. The CFTR gene is highly expressed in the pancreas, and cystic fibrosis patients with only mild variants have a $40-80 \%$ increased risk of developing chronic pancreatitis [39]. While a number of mouse models have been developed to study cystic fibrosis, the most well studied in relation to pancreatic function are mice expressing a S489X mutation in the Cftr which results in the formation of a truncated protein [40-42]. As described in the original study by Snouwaert et al. [42], these mice show features common to that of young cystic fibrosis patients, with later publications describing in detail a mild form of pancreatitis characterised by acinar dysfunction and altered pancreatic digestive enzymes [40, 41, 43]. However, these mice do not develop high levels of inflammation or pancreatic fibrosis suggesting that chronic pancreatitis does not develop, and multi-organ cystic fibrosis makes it a difficult model to use specifically for the purpose of studying pancreatitis.

Keratin genes control epithelia-specific intermediatefilament (IF) proteins and mutations in the K8 and K18 isoforms, which are expressed in simple or single-layered epithelial tissues, increase susceptibility to inflammatory liver diseases $[44,45]$. The forced overexpression of these proteins in mice leads to a progressive age-dependent decline in pancreatic exocrine function, alterations in acinar cell morphology, and eventually marked pancreatic fibrosis with inflammation and lipid infiltration by $1-2$ year of age [46, 47]. Interestingly, Casanova et al. [46] noted that mice expressing mutant $\mathrm{Tg} f b$ type II (a model where loss of TGF- $\beta$ signalling control over acinar growth result in pancreatitis) also have greatly elevated levels of K8/18 in pancreata. This potentially suggests a model where growth factor/cytokine signaling responses drive pancreatitis through altering epithelial function.

In support of these observations, defects in epithelial cell cilia is thought to play a central role in the development of polycystic kidney disease (PKD), an inherited disorder where renal tubule epithelium becomes structurally abnormal and resulting in the development of cysts. The incidence of pancreatitis is increased in patients with PKD, however it is still rare in pancreatitis patients and normally limited to those with pancreatic duct compression [48, 49]. Two murine models of PKD have been shown to present with pancreatitis pathophysiology. Zang et al. [50] showed that previously described mice with a PKD phenotype induced through the loss of polaris ( $T g 737)$ [51], an transport protein required for ciliogenesis, have abnormal endocrine and exocrine functions of the pancreas. These mice show disorganised and apoptotic acini, fibrosis, and glucose intolerance without immune cell infiltration, suggesting this model does not follow the traditional inflammatory pathway of pancreatitis development. In contrast, pancreatic epithelia specific ablation of the Kif3a gene, which is also essential for cilia formation, results in progressive acinar-to-ductal metaplasia, fibrosis, and replacement of acinar cells with adipose tissue [52]. Furthermore, the pancreatic phenotype is lost when Kif3a deletion is induced in late development (4 weeks), suggesting it is the result of loss of embryotic cilia development rather than an inducible pancreatitis. While transgenic mouse lines may present with changes of pancreatic diseases associated with PKD, whether they are truly representative of human pancreatitis is debatable.

\section{Inflammation}

In support of the growing evidence that cytokine signalling, through their roles as growth factors or inflammatory mediators, can play a key role in regulating pancreatic 
cell function in humans, genetic manipulation of various cytokines, cytokine receptors, or downstream target pathways in mice have now been reported to promote the development of pancreatitis (Table 1). Since inflammatory processes are often coupled with endoplasmic reticulum (ER) stress and autophagy, it is difficult to distinguish which is the driving factors. While there are a number of models available (Table 1), below we summarise those that appear to be most representative of the human disease.

Transforming growth factor- $\beta$ (TGF- $\beta$ ) is a super-group of secreted cytokines that binds to multiple cell surface receptors leading to the phosphorylation activation of cellular signalling events that control an array of cellular process either directly or through Smad transcription factor-regulated gene expression. TGF- $\beta$ plays a central role in both normal development of the pancreas and disease. Mouse models that overexpress $\operatorname{Tg} f-\beta 1$ specifically in the pancreas [53-55] show evidence of pancreatitis development from birth with progressive development of pancreatic fibrosis and pronounced immune cell infiltration by 2 weeks of age, and eventual loss of acinar cells. Despite the mice appear healthy, concurrent overexpression of the pro-inflammatory cytokine TNF $\alpha$ in pancreatic $\beta$-cells promotes the development of diabetes with mice showing pronounced hyperglycemia by 4 month [54]. This is a phenotype that is not seen in either of the individual mouse strains and highlights multifaceted nature of pancreatitis, potentially suggesting that the ideal GEMM of pancreatitis may target multiple pathways. To further illustrate the complex signalling processes controlling pancreatic function, not only does overexpression of $\mathrm{Tg} f b$ promote pancreatitis but a similar phenotype is seen in mice expressing a dominate negative $\mathrm{Tgfb}$ receptor II (inactive) in epithelia cells [46] or when $\mathrm{Tg} f b$ receptor II is knockout exclusively in dendritic cells [56]. This suggests that there is a hormetic response to TGF- $\beta$ signalling in the pancreatic cells where too much or too little can result in disease development.

Similar to TGF- $\beta$, the cystokines IL-b and lymphotoxin-b signal through their respective receptors to induce inflammatory responses. These inflammatory responsesaremediated, at least in part, by IkBkinase(IKK), an enzyme that phosphorylates the inhibitory protein I $\mathrm{B} \alpha$ resulting in the release of NFkb which translocate to the cell's nucleus and regulates inflammatory gene expression. These pathways may be upregulated in postpancreatitis diabetes and, hence, hold a considerable translational potential [57-59].

Indeed, overexpression or constitutiveactivation of $I l b$, lymphotoxin-b or Ikkb [60-62] specifically in the pancreas results in the development of a form of pancreatitis that shows many inflammatory related hallmarks of human pancreatitis, including an increase in circulating cytokines and/or pancreatic gene expression such as $T N F-\alpha, I L-\beta, I L-6, C X C L$ 's, and invasion of inflammatory cells in acinar cells. Furthermore, the lymphotoxin-b overexpression model showed impairments in insulin sensitivity and alterations in pancreatic lipid metabolism, manifestations that are increasingly being associated with pancreatitis [62]. This was associated with two phases of disease development, early onset pancreatitis initiating an innate immune response followed by recruitment of lymphocytes to the damaged pancreas, exacerbating the immune response leading to systemic inflammation.

Consistent with the cytokine signalling-IKK-NFkbinflammation pathway in the development of pancreatitis, both genetic suppression of $I k k b$ or pharmacological inhibition of $N f k b$ have shown promise in protecting from caerulein-induced pancreatitis, and constitutive activation of $I k k b$ in acinar cells is sufficient to induce acute pancreatitis [61, 63]. In contrast, however, the genetic disruption of $I k k a$ similarly leads to disruption of autophagy processes and the induction of ER stress cumulating in pancreatic fibrosis, inflammation, and the release of pancreatic enzymes into circulation [64]. This highlights the contrasting role of the IKK-NFkb pathway in maintaining pancreatic integrity, where both excessive and insufficient activation can cause pancreatitis.

\section{ER stress and autophagy}

The pancreas is responsible for producing large amounts of secretory-proteins, rendering it highly susceptible to protein misfolding responses. One prominent hypothesis in the aetiology of chronic pancreatitis is that there is a cyclical relationship between ER stress and protein misfolding, which initiates the unfolded protein response in an effort to restore proteostasis. However, if this is not achieved further ER stress is induced, leading to inflammatory responses and apoptosis [65, 66]. Indeed, ER stress associated proteins are upregulated in the pancreatic islets of patients with pancreatitis $[67,68]$. While ER stress is induced by a variety of both physiological/pathological perturbations as well as chemical or mechanical stress, the primary pathway targeted genetically to initiate ER stress to study its role on pancreatitis is either the globally $[69,70]$ or pancreatic acinar cell specific knockout of Perk. Perk is a transmembrane protein of the ER and acts via eukaryotic initiation factor 2a (eIF-2a) to couple protein folding to polypeptide biosynthesis, and its ablation elevates ER 
stress [71]. Early studies observed that global ablation of Perk resulted in pancreatic islet apoptosis and impairment of glucose homeostasis at a young age, coupled with growth retardation and high rates of mortality. This appears to be a very severe phenotype perhaps not wholly representative of the disease in humans, and is likely a result of tissue-cross talk resulting from the global Perk ablation methodology. Indeed, when Perk in disrupted specifically in acinar cells, a much milder degree of acinar cell death is observed and this is associated with both inflammation and lipid infiltration [72], potentially better replicating the pathogenesis seen in humans.

Acinar cells are responsible for very high rates of protein synthesis for secretion. This makes them highly susceptible to accumulating misfolded proteins, which if not degraded then can induce ER stress and an associated inflammatory response. As such, insufficient autophagy has been hypothesised to contribute to the development of pancreatitis [73]. Recently, several mouse models that lack key autophagy regulating genes have been developed and investigated for their susceptibility to pancreatitis. Liposomal degradation of cytoplasmic material is the final step in autophagy, and disrupting this process by global knockout of the lysosome-associated membrane proteins 2 (Lamp-2) leads to vacuolization of acinar due to impaired autophagic flux leading to both inflammation and necrosis of the pancreas [74]. Consistent with this, samples from human pancreatitis patients show reduced Lamp-2) expression [74]. While this highlights the important role of liposomal regulation in maintaining pancreatic homeostasis, whether it is a cause or an effect of pancreatitis is unclear. It is a somewhat crude model given the integral role of liposomes in maintaining cellular homeostasis. An approach that more specifically targets autophagy is the pancreatic specific deletion of the key autophagy regulatory genes Atg5 and Atg7 [75-77]. Both of these knockout models show progressive development of pancreatic atrophy, fibrosis, necrosis and inflammation. Some systemic effects were also noted with the Atg7 knockout mice showing increased $\alpha$-amylase and lipase, impaired glucose tolerance and reduced lifespan $[75,77]$. In addition to showing many of the characteristics of human pancreatitis, these models have begun to provide further insight into potential underlying mechanisms. The reduced autophagy response and increased ER stress was associated with mitochondrial dysfunction $[75,77]$, potentially altering intracellular energy balance and elevating reactive oxygen species, further promoting cell damage. Indeed, this supports observations the

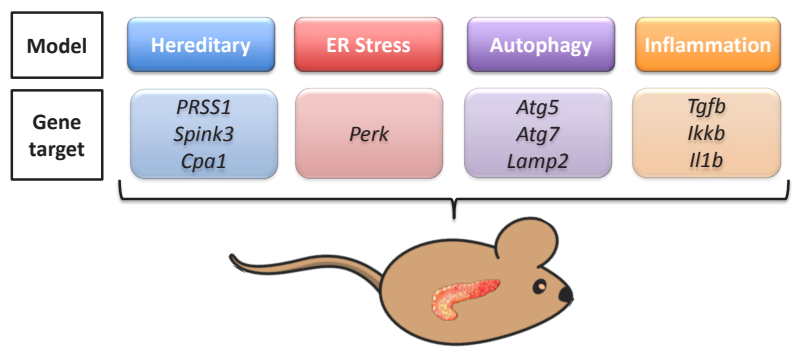

Figure 1: Summary of common genetically engineered mouse models of pancreatitis

exogenous antioxidant supplements may attenuate pain associated with chronic pancreatitis [78, 79] and reduce hospital stays and complications associated with acute pancreatitis [80].

\section{Summary}

In order to develop more effective treatment approaches for pancreatitis it is important to better understand the underlying pathophysiology. Genetic mouse strains may offer unique insights into the mechanisms governing the development and progression of pancreatitis, however it is important to select the most appropriate model (Figure 1). There are a number of different GEMM that show spontaneous development of pancreatitis, and those that target genes involved in the regulation of ER stress and autophagy (such as Perk, lymphotoxin-b, and Atg5/7) appear to best replicate non-genetic pancreatitis. While popular models that currently represent pancreatitis that has a strong genetic component are those that target the SPINK1 or PRSS1 genes, their usefulness may be limited as they have roles in development and potential toxic effects when the human PRSS1 gene is expressed in mice. Newly developed models, such as the N256K mutation in Cpa1, may provide novel insights.

Acknowledgements: TLM and MSP are supported by Rutherford Discovery Fellowships (Royal Society of New Zealand).

Competing Financial Interests: The authors declare no competing financial interests

Ethics approval and consent to Participate: Not applicable 


\section{References}

1. Xiao, A.Y., et al., Global incidence and mortality of pancreatic diseases: a systematic review, meta-analysis, and metaregression of population-based cohort studies. Lancet Gastroenterol Hepatol, 2016. 1(1): p. 45-55.

2. Sankaran, S.J., et al., Frequency of progression from acute to chronic pancreatitis and risk factors: a meta-analysis. Gastroenterology, 2015. 149(6): p. 1490-1500 e1.

3. Jivanji, C.J., et al., New-Onset Diabetes After Acute and Critical Illness: A Systematic Review. Mayo Clin Proc, 2017. 92(5): p. 762-773.

4. Pendharkar, S.A., J. Mathew, and M.S. Petrov, Age- and sexspecific prevalence of diabetes associated with diseases of the exocrine pancreas: A population-based study. Dig Liver Dis, 2017. 49(5): p. 540-544.

5. Petrov, M.S., Diabetes of the exocrine pancreas: American Diabetes Association-compliant lexicon. Pancreatology, 2017. 17(4): p. 523-526.

6. Singh, R.G., et al., Ectopic fat accumulation in the pancreas and its clinical relevance: $A$ systematic review, meta-analysis, and meta-regression. Metabolism, 2017. 69: p. 1-13.

7. Petrov, M.S. and J.A. Windsor, Conceptual framework for classifying the severity of acute pancreatitis. Clin Res Hepatol Gastroenterol, 2012. 36(4): p. 341-4.

8. Dellinger, E.P., et al., Determinant-based classification of acute pancreatitis severity: an international multidisciplinary consultation. Ann Surg, 2012. 256(6): p. 875-80.

9. Yang, C.J., et al., Predictors of severe and critical acute pancreatitis: a systematic review. Dig Liver Dis, 2014. 46(5): p. 446-51.

10. Stigliano, S., et al., Early management of acute pancreatitis: $A$ review of the best evidence. Dig Liver Dis, 2017. 49(6): p. 585594.

11. Halonen, K.I., et al., Multiple organ dysfunction associated with severe acute pancreatitis. Crit Care Med, 2002. 30(6): p. 1274-9.

12. Mofidi, R., et al., Association between early systemic inflammatory response, severity of multiorgan dysfunction and death in acute pancreatitis. Br J Surg, 2006. 93(6): p. 738-44.

13. Lerch, M.M. and F.S. Gorelick, Models of acute and chronic pancreatitis. Gastroenterology, 2013. 144(6): p. 1180-93.

14. Reed, A.M., et al., Low $\mathrm{pH}$ enhances connexin32 degradation in the pancreatic acinar cell. Am J Physiol Gastrointest Liver Physiol, 2014. 307(1): p. G24-32.

15. Ji, B., et al., Human pancreatic acinar cells lack functional responses to cholecystokinin and gastrin. Gastroenterology, 2001. 121(6): p. 1380-90.

16. Klauss, S., et al., Genetically induced vs. classical animal models of chronic pancreatitis: a critical comparison. FASEB J, 2018: p. fj201800241RR.

17. Magnuson, M.A. and A.B. Osipovich, Pancreas-specific Cre driver lines and considerations for their prudent use. Cell Metab, 2013. 18(1): p. 9-20.

18. Feil, R., et al., Ligand-activated site-specific recombination in mice. Proc Natl Acad Sci U S A, 1996. 93(20): p. 10887-90.

19. Whitcomb, D.C., et al., Hereditary pancreatitis is caused by a mutation in the cationic trypsinogen gene. Nat Genet, 1996. 14(2): p. 141-5.
20. Gorry, M.C., et al., Mutations in the cationic trypsinogen gene are associated with recurrent acute and chronic pancreatitis. Gastroenterology, 1997. 113(4): p. 1063-8.

21. Archer, H., et al., A mouse model of hereditary pancreatitis generated by transgenic expression of $\mathrm{R} 122 \mathrm{H}$ trypsinogen. Gastroenterology, 2006. 131(6): p. 1844-55.

22. Athwal, T., et al., Expression of human cationic trypsinogen (PRSS1) in murine acinar cells promotes pancreatitis and apoptotic cell death. Cell Death Dis, 2014. 5: p. e1165.

23. Gaiser, S., et al., Intracellular activation of trypsinogen in transgenic mice induces acute but not chronic pancreatitis. Gut, 2011. 60(10): p. 1379-88.

24. Hegyi, E. and M. Sahin-Toth, Genetic Risk in Chronic Pancreatitis: The Trypsin-Dependent Pathway. Dig Dis Sci, 2017. 62(7): p. 1692-1701.

25. Witt, H., et al., Mutations in the gene encoding the serine protease inhibitor, Kazal type 1 are associated with chronic pancreatitis. Nat Genet, 2000. 25(2): p. 213-6.

26. Awano, H., et al., Childhood-onset hereditary pancreatitis with mutations in the CT gene and SPINK1 gene. Pediatr Int, 2013. 55(5): p. 646-9.

27. Ohmuraya, M., et al., Autophagic cell death of pancreatic acinar cells in serine protease inhibitor Kazal type 3-deficient mice. Gastroenterology, 2005. 129(2): p. 696-705.

28. Romac, J.M., et al., Transgenic expression of pancreatic secretory trypsin inhibitor-1 rescues SPINK3-deficient mice and restores a normal pancreatic phenotype. Am J Physiol Gastrointest Liver Physiol, 2010. 298(4): p. G518-24.

29. Witt, H., et al., Variants in CPA1 are strongly associated with early onset chronic pancreatitis. Nat Genet, 2013. 45(10): p. 1216-20.

30. Hegyi, E. and M. Sahin-Toth, Human CPA1 mutation causes digestive enzyme misfolding and chronic pancreatitis in mice. Gut, 2018.

31. Singh, R.G., et al., Associations between circulating levels of adipocytokines and abdominal adiposity in patients after acute pancreatitis. Clin Exp Med, 2017. 17(4): p. 477-487.

32. Singh, R.G., et al., Abdominal obesity and insulin resistance after an episode of acute pancreatitis. Dig Liver Dis 2018;50:1081-1087.

33. Durno, C., et al., Genotype and phenotype correlations in patients with cystic fibrosis and pancreatitis. Gastroenterology, 2002. 123(6): p. 1857-64.

34. Cohn, J.A. and P.S. Jowell, Are mutations in the cystic fibrosis gene important in chronic pancreatitis? Surg Clin North Am, 1999. 79(4): p. 723-31, viii.

35. Choudari, C.P., et al., Risk of pancreatitis with mutation of the cystic fibrosis gene. Am J Gastroenterol, 2004. 99(7): p. 135863.

36. Yazdanpanah, K., et al., Recurrent acute pancreatitis and cholangitis in a patient with autosomal dominant polycystic kidney disease. Int J Prev Med, 2013. 4(2): p. 233-6.

37. Cavestro, G.M., et al., Association of keratin 8 gene mutation with chronic pancreatitis. Dig Liver Dis, 2003. 35(6): p. 416-20.

38. Treiber, M., et al., Keratin 8 sequence variants in patients with pancreatitis and pancreatic cancer. J Mol Med (Berl), 2006. 84(12): p. 1015-22.

39. Ooi, C.Y., et al., Type of CFTR mutation determines risk of pancreatitis in patients with cystic fibrosis. Gastroenterology, 2011. 140(1): p. 153-61. 
40. De Lisle, R.C., Increased expression of sulfated gp300 and acinar tissue pathology in pancreas of CFTR(-/-) mice. Am J Physiol, 1995. 268(4 Pt 1): p. G717-23.

41. Durie, P.R., et al., Characteristic multiorgan pathology of cystic fibrosis in a long-living cystic fibrosis transmembrane regulator knockout murine model. Am J Pathol, 2004. 164(4): p. 1481-93.

42. Snouwaert, J.N., et al., An animal model for cystic fibrosis made by gene targeting. Science, 1992. 257(5073): p. 1083-8.

43. Kaur, S., et al., Acidic duodenal pH alters gene expression in the cystic fibrosis mouse pancreas. Am J Physiol Gastrointest Liver Physiol, 2004. 287(2): p. G480-90.

44. Ku, N.O., et al., Keratins let liver live: Mutations predispose to liver disease and crosslinking generates Mallory-Denk bodies. Hepatology, 2007. 46(5): p. 1639-49.

45. Uitto, J., G. Richard, and J.A. McGrath, Diseases of epidermal keratins and their linker proteins. Exp Cell Res, 2007. 313(10): p. 1995-2009.

46. Casanova, M.L., et al., Exocrine pancreatic disorders in transsgenic mice expressing human keratin 8. J Clin Invest, 1999. 103(11): p. 1587-95.

47. Toivola, D.M., et al., Keratin overexpression levels correlate with the extent of spontaneous pancreatic injury. Am J Pathol, 2008. 172(4): p. 882-92.

48. Torra, R., et al., Ultrasonographic study of pancreatic cysts in autosomal dominant polycystic kidney disease. Clin Nephrol, 1997. 47(1): p. 19-22.

49. Mikolajczyk, A.E., H.S. Te, and A.B. Chapman, Gastrointestinal Manifestations of Autosomal-Dominant Polycystic Kidney Disease. Clin Gastroenterol Hepatol, 2017. 15(1): p. 17-24.

50. Zhang, Q., et al., Disruption of IFT results in both exocrine and endocrine abnormalities in the pancreas of Tg737(orpk) mutant mice. Lab Invest, 2005. 85(1): p. 45-64.

51. Moyer, J.H., et al., Candidate gene associated with a mutation causing recessive polycystic kidney disease in mice. Science, 1994. 264(5163): p. 1329-33.

52. Cano, D.A., S. Sekine, and M. Hebrok, Primary cilia deletion in pancreatic epithelial cells results in cyst formation and pancreatitis. Gastroenterology, 2006. 131(6): p. 1856-69.

53. Vogelmann, R., et al., Effects of fibrogenic mediators on the development of pancreatic fibrosis in a TGF-beta1 transgenic mouse model. Am J Physiol Gastrointest Liver Physiol, 2001. 280(1): p. G164-72.

54. Sanvito, F., et al., TGF-beta 1 overexpression in murine pancreas induces chronic pancreatitis and, together with TNFalpha, triggers insulin-dependent diabetes. Biochem Biophys Res Commun, 1995. 217(3): p. 1279-86.

55. Lee, M.S., et al., Accumulation of extracellular matrix and developmental dysregulation in the pancreas by transgenic production of transforming growth factor-beta 1. Am J Pathol, 1995. 147(1): p. 42-52.

56. Boomershine, C.S., et al., Autoimmune pancreatitis results from loss of TGFbeta signalling in S100A4-positive dendritic cells. Gut, 2009. 58(9): p. 1267-74.

57. Gillies, N., et al., Interleukin- 6 is associated with chronic hyperglycemia and insulin resistance in patients after acute pancreatitis. Pancreatology, 2016. 16(5): p. 748-55.

58. Pendharkar, S.A., et al., Pro-inflammatory cytokines after an episode of acute pancreatitis: associations with fasting gut hormone profile. Inflamm Res, 2018. 67(4): p. 339-350.
59. Gillies, N.A., et al., Fasting levels of insulin and amylin after acute pancreatitis are associated with pro-inflammatory cytokines. Arch Physiol Biochem, 2017. 123(4): p. 238-248.

60. Colby, J.K., et al., Progressive metaplastic and dysplastic changes in mouse pancreas induced by cyclooxygenase- 2 overexpression. Neoplasia, 2008. 10(8): p. 782-96.

61. Baumann, B., et al., Constitutive IKK2 activation in acinar cells is sufficient to induce pancreatitis in vivo. J Clin Invest, 2007. 117(6): p. 1502-13.

62. Seleznik, G.M., et al., Lymphotoxin beta receptor signaling promotes development of autoimmune pancreatitis. Gastroenterology, 2012. 143(5): p. 1361-74.

63. Ethridge, R.T., et al., Selective inhibition of NF-kappaB attenuates the severity of cerulein-induced acute pancreatitis. J Am Coll Surg, 2002. 195(4): p. 497-505.

64. Li, N., et al., Loss of acinar cell IKKalpha triggers spontaneous pancreatitis in mice. J Clin Invest, 2013. 123(5): p. 2231-43.

65. Sahin-Toth, M., Genetic risk in chronic pancreatitis: the misfolding-dependent pathway. Curr Opin Gastroenterol, 2017. 33(5): p. 390-395.

66. Lugea, A., R.T. Waldron, and S.J. Pandol, Pancreatic adaptive responses in alcohol abuse: Role of the unfolded protein response. Pancreatology, 2015. 15(4 Suppl): p. S1-5.

67. Sah, R.P., et al., Endoplasmic reticulum stress is chronically activated in chronic pancreatitis. J Biol Chem, 2014. 289(40): p. 27551-61.

68. Petrov, M.S., Therapeutic implications of oxidative stress in acute and chronic pancreatitis. Curr Opin Clin Nutr Metab Care, 2010. 13(5): p. 562-8.

69. Harding, H.P., et al., Diabetes mellitus and exocrine pancreatic dysfunction in perk-/- mice reveals a role for translational control in secretory cell survival. Mol Cell, 2001. 7(6): p. 115363.

70. Zhang, P., et al., The PERK eukaryotic initiation factor 2 alpha kinase is required for the development of the skeletal system, postnatal growth, and the function and viability of the pancreas. Mol Cell Biol, 2002. 22(11): p. 3864-74.

71. Harding, H.P., et al., Perk is essential for translational regulation and cell survival during the unfolded protein response. Mol Cell, 2000. 5(5): p. 897-904.

72. lida, K., et al., PERK eIF2 alpha kinase is required to regulate the viability of the exocrine pancreas in mice. BMC Cell Biol, 2007. 8: p. 38.

73. Gukovsky, l., et al., Inflammation, autophagy, and obesity: common features in the pathogenesis of pancreatitis and pancreatic cancer. Gastroenterology, 2013. 144(6): p. 1199-209 e4.

74. Mareninova, O.A., et al., Lysosome associated membrane proteins maintain pancreatic acinar cell homeostasis: LAMP-2 deficient mice develop pancreatitis. Cell Mol Gastroenterol Hepatol, 2015. 1(6): p. 678-694.

75. Antonucci, L., et al., Basal autophagy maintains pancreatic acinar cell homeostasis and protein synthesis and prevents $E R$ stress. Proc Natl Acad Sci U S A, 2015. 112(45): p. E6166-74.

76. Diakopoulos, K.N., et al., Impaired autophagy induces chronic atrophic pancreatitis in mice via sex- and nutrition-dependent processes. Gastroenterology, 2015. 148(3): p. 626-638 e17.

77. Zhou, X., et al., RIP3 attenuates the pancreatic damage induced by deletion of ATG7. Cell Death Dis, 2017. 8(7): p. e2918. 
78. Kirk, G.R., et al., Combined antioxidant therapy reduces pain and improves quality of life in chronic pancreatitis. I Gastrointest Surg, 2006. 10(4): p. 499-503.

79. Bhardwaj, P., et al., A randomized controlled trial of antioxidant supplementation for pain relief in patients with chronic pancreatitis. Gastroenterology, 2009. 136(1): p. 149-159 e2.

80. Sateesh, J., et al., Effect of antioxidant therapy on hospital stay and complications in patients with early acute pancreatitis: $a$ randomised controlled trial. Trop Gastroenterol, 2009. 30(4): p. 201-6.

81. Dimagno, M.J., et al., A proinflammatory, antiapoptotic phenotype underlies the susceptibility to acute pancreatitis in cystic fibrosis transmembrane regulator (-/-) mice. Gastroenterology, 2005. 129(2): p. 665-81.

82. Freedman, S.D., H.F. Kern, and G.A. Scheele, Pancreatic acinar cell dysfunction in CFTR(-/-) mice is associated with impairments in luminal $\mathrm{pH}$ and endocytosis. Gastroenterology, 2001. 121(4): p. 950-7.

83. Wang, G., et al., Ablation of huntingtin in adult neurons is nondeleterious but its depletion in young mice causes acute pancreatitis. Proc Natl Acad Sci U S A, 2016. 113(12): p. 3359 64.

84. Tanaka, Y., et al., Accumulation of autophagic vacuoles and cardiomyopathy in LAMP-2-deficient mice. Nature, 2000. 406(6798): p. 902-6.

85. Marrache, F., et al., Overexpression of interleukin-1beta in the murine pancreas results in chronic pancreatitis. Gastroenterology, 2008. 135(4): p. 1277-87.
86. Kurisaki, H., et al., Autoimmune gastro-pancreatitis with antiprotein disulfide isomerase-associated 2 autoantibody in Aire deficient BALB/cAnN mice. PLoS One, 2013. 8(8): p. e73862.

87. Iglesias, A., et al., Diabetes and exocrine pancreatic insufficiency in E2F1/E2F2 double-mutant mice. J Clin Invest, 2004. 113(10): p. 1398-407.

88. Dessimoz, J., et al., Pancreas-specific deletion of beta-catenin reveals Wnt-dependent and Wnt-independent functions during development. Curr Biol, 2005. 15(18): p. 1677-83.

89. Bombardelli, L., et al., Pancreas-specific ablation of beta1 integrin induces tissue degeneration by disrupting acinar cell polarity. Gastroenterology, 2010. 138(7): p. 2531-40, 2540 e1-4.

90. Lunova, M., et al., Hepcidin knockout mice spontaneously develop chronic pancreatitis owing to cytoplasmic iron overload in acinar cells. J Pathol, 2017. 241(1): p. 104-114.

91. Ji, B., et al., Ras activity levels control the development of pancreatic diseases. Gastroenterology, 2009. 137(3): p. 107282, 1082 e1-6.

92. Gabbi, C., et al., Pancreatic exocrine insufficiency in LXRbeta-/mice is associated with a reduction in aquaporin-1 expression. Proc Natl Acad Sci U S A, 2008. 105(39): p. 15052-7.

93. Miralles, F., et al., Conditional inactivation of the murine serum response factor in the pancreas leads to severe pancreatitis. Lab Invest, 2006. 86(10): p. 1020-36. 